\title{
An Experimental Study on the Sediment Distribution from the Spraying of Pesticide Solution by High-efficiency, Wide-coverage and Long-range Power Sprayer during the Later Stage of Rice Growth
}

\author{
Gong Yan ${ }^{1, \text { a , }}$, Fu Ximin ${ }^{1}$, Wang Guo ${ }^{1}$, Zhang Xiao ${ }^{1}$, Chen Xiao ${ }^{1}$, Liu Dejiang ${ }^{1}$ \\ ${ }^{1}$ Nanjing Research Institute for Agricultural Mechanization Ministry of Agriculture P. R. China, \\ Nanjing, 210014, China \\ ajsgymm@hotmail.com
}

Keywords: Sprayer, Spray Gun, Rice, Drift Loss, Sediment Distribution

\begin{abstract}
This experiment used high-efficiency, wide-coverage and long-range power sprayers to spray pesticide solution during the later stage of rice growth. It measured the quantity of pesticide sediment, then analyzed and studied the distribution pattern of pesticide sediment on the rice plants and the sediment ratios in the rice field. This experimental study indicates: spraying pesticide solution results in relatively high sediment distribution ratios; the upper parts of the rice plants are most able to receive the pesticide solution; this new sprayer model sprays evenly and penetrates well, effectively improving sediment quantity of pesticide solution on the middle and lower parts of the rice while reducing pesticide quantity.
\end{abstract}

\section{Introduction}

The high-efficiency, wide-coverage and long-range power sprayer is a new type of plant protection machine for disease and pest control and prevention in large, medium, and small paddies ${ }^{1}$. Meanwhile, it can be used for disease and pest control in large-field crops, fruit orchards and gardens where water supply is convenient. This series of sprayers has obvious advantages such as high operating pressure, smooth and reliable operation, long spray range, even pesticide spraying, high efficiency, and low labor intensiveness. Using high-pressure, wide-coverage and long-range even spraying, this machine enhances the penetration of spray droplets and improves disease and pest control results for the middle and lower parts of the rice plants. It uses a series of high-efficiency, wide-coverage and long-range multi-nozzle combination spray guns, which combine various nozzle models such as the long-range spray, the conical spray, and the fan-shaped spray so that the spray droplets from the spray gun are evenly distributed along the spray trajectory. This feature overcomes the original spray gun's shortcomings such as big spray droplets, uneven distribution, and narrow spray width, thereby significantly improving operating efficiency through its usage. In addition, it avoids repeated and missed spraying in the prevention and control of paddy field operations and improves the pesticide's utilization rate ${ }^{2}$. It increases work efficiency, reduces labor intensiveness, and ensures the safety of pesticide application without having to go into the paddy field, representing the ideal model for achieving mechanized disease and pest control of rice crops in China.

\section{The Wide-coverage, Long-range Performance Design of the Sprayer}

Rice plants become leafy during its later stage of growth, which makes it very difficult to apply pesticide solution to the middle and lower parts of the plants through regular spraying methods. Spraying by dousing uses large quantities of pesticide and applies unevenly, producing relatively poor prevention results 3 . This has always been an obstacle in the pest control and prevention of rice.

Using Wide-Coverage, Long-Range Multi-Nozzle Combination Spray Guns to Achieve Uniformity in Spray Quantity Along the Trajectory. High-efficiency, wide-coverage and 
long-range multi-nozzle combination spray gun combines various nozzle models including the long-range spray, the conical spray and the fan-shaped spray to enable the spray gun to distribute uniformly droplets along the spray trajectory. The coefficient of variation of the spray distribution's uniformity is lower than 0.35 , thus overcoming disadvantages such as the large size of spray droplets, unevenness, and narrow spray width and significantly improving operating efficiency. See Fig. 1 and Fig. 2 for the spray quantity distribution of the wide-coverage, long-distance, multi-nozzle combination spray gun.

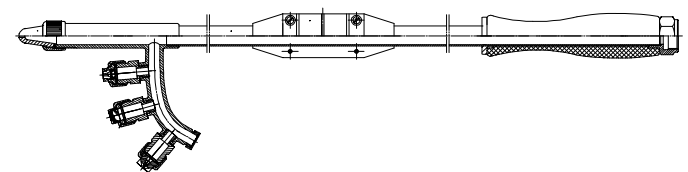

Fig. 1 Structural diagram of the wide-coverage, long-range multi-nozzle combination spray gun. Combination nozzle spray quantity distribution

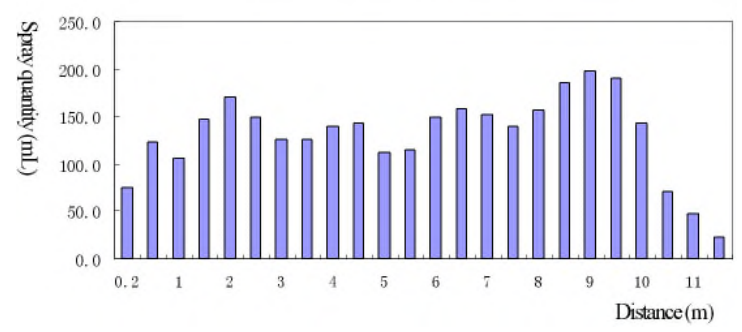

Fig. 2 Diagram of the spray quantity distribution of the wide-coverage, long-range multi-nozzle combination spray gun

Using High-Pressure Plunger Pump, Pressure-Resistant Spray Hose and Optimized Small Power Configuration to Achieve Wide-Coverage and Long-Range Even Spraying. The use of high-pressure spray increases the penetration of spray droplets, which significantly enhances the prevention and control results for the middle and lower parts of rice plants in their later growth state. The application experiment on nearly 20,000 mu (Chinese acre) of paddy field indicates: the effective spraying width of the spray gun $>12-18 \mathrm{~m}$, the applied pesticide quantity $<30 \mathrm{~L} / \mathrm{mu}$, the operating efficiency $>20-40 \mathrm{mu} /$ hour, the operational reliability coefficient $>96.5 \%$, and the water and pesticide conservation rate $>30 \%$. See Figure 3 for application results in the paddy field.

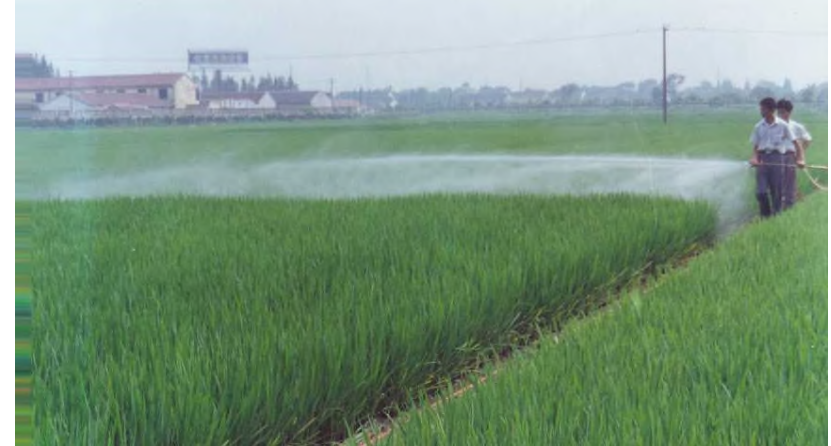

Fig. 3 Application results of the high-efficiently, wide-coverage and long-range power sprayer in the rice paddy

\section{Field Test on Sediment Distribution of Pesticide Solution Sprayed on Rice during Its Later Growth Stage}

Using the "Lichunhong-2R" aqueous tracer agent as test solution, this field test employed the high-efficiency, wide-coverage and long-range power sprayer configured with long-range combination spray guns of various flow rates. It measured the sediment quantity of pesticide solution on upper, middle, and lower parts of the rice plants as well as on the ground. Furthermore, it tested and studied the characteristics and patterns of the sediment distribution, and analyzed 
whether the sediment distribution of pesticide solution enhanced the disease and pest prevention and control of rice in its later growth stage. Lastly, it studied and analyzed the downward penetrability of spray droplets in the final stage of rice growth, with the long-range combination spray guns of various flow rates operating under certain spraying pressures.

\section{Experiment Conditions.}

Experiment location. The location chosen for the experiment was the Xinyang Experiment Station of the Jiangsu Coastland Agricultural Sciences Research Institute, located in the east of Yancheng City. The station has over $533 \mathrm{hm}^{2}$ of cultivated land, of which rice paddies occupy more than $133 \mathrm{hm}^{2}$. The rice paddies are organized, with the horizontal and vertical irrigation channels intersecting to form a net with the dimensions of 50m x 300m and the platform fields running in the north-south direction. The conditions for mechanized operation are fairly good.

Crops used in experiment. The rice crop used in the experiment was "Wuyugeng-No. 3.".All of the rice paddies were fertile beds in dry conditions and manually transplanted at equal row spacing. Plant spacing was $14 \mathrm{~cm} \times 18 \mathrm{~cm}$, planting density was approximately $1,333 \mathrm{holes} / \mathrm{hm}^{2}$ and the average height of plants in the experiment was $81 \mathrm{~cm}$.

Sprayers used in experiment. One set of High-efficiency, Wide-coverage and Long-range Power Spray 3WKY-40, with the following configurations:

High-efficiency, Wide-coverage and Long-range Combination Spray Gun PQ20, with nozzle flow rate calibrated at $18 \mathrm{~L} / \mathrm{min}$;

High-efficiency, Wide-coverage and Long-range Combination Spray Gun PQ30, with nozzle flow rate calibrated at $26 \mathrm{~L} / \mathrm{min}$;

High-efficiency, Wide-coverage and Long-range Combination Spray gun PQ400, with nozzle flow rate calibrated at $36 \mathrm{~L} / \mathrm{min}$.

Pesticide solution used in experiment. The solution used in the experiment was the "Lichunhong-2R" aqueous tracer agent, a biological dye freely soluble in water with good photolysis stability. This aqueous solution could be easily washed off of the targets after spraying. The concentration of the pesticide solution prepared in the experiment was 4000ppm.

Field partitioning in experiment. The field used for the experiment was partitioned as follows:

The experimental field area for PQ20 spray gun: $12 \mathrm{~m} X 13 \mathrm{~m}=156 \mathrm{~m}^{2}$

The experimental field area for PQ30 spray gun: $15 \mathrm{~m} X 13 \mathrm{~m}=195 \mathrm{~m}^{2}$

The experimental field area for PQ 40 spray gun: $17 \mathrm{~m} \mathrm{X} 13 \mathrm{~m}=221 \mathrm{~m}^{2}$

Note: The field lengths, $12 \mathrm{~m}, 15 \mathrm{~m}$, and $17 \mathrm{~m}$, respectively correspond to the ranges of the PQ20, PQ30, and PQ40 spray guns. The width of $13 \mathrm{~m}$ is the combined width of the three ridges; each ridge (path included) is approximately $4.2 \mathrm{~m}$.

The walking direction of the spray and the sampling area are respectively shown in Fig. 4.

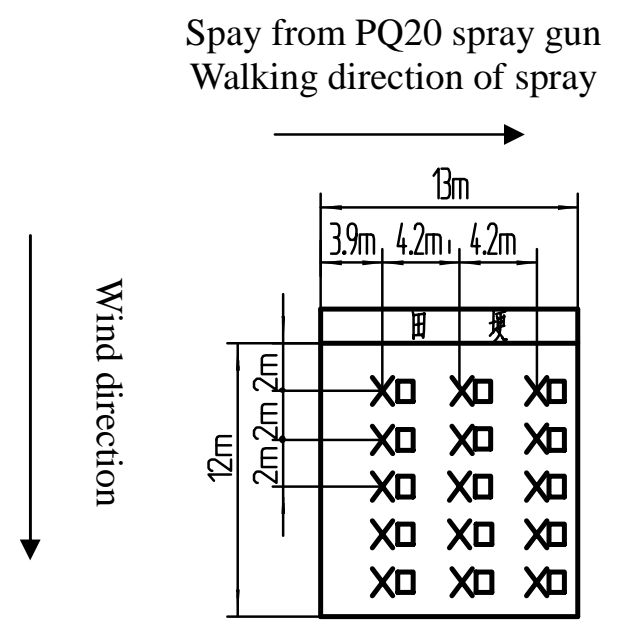

Spray from PQ30 spray gun Walking direction of spray

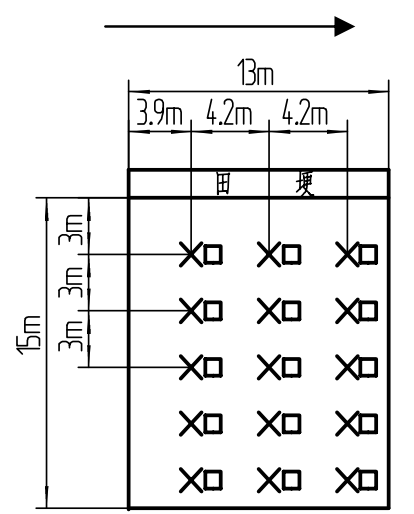

Spray from PQ40 spray gun Walking direction of Spray

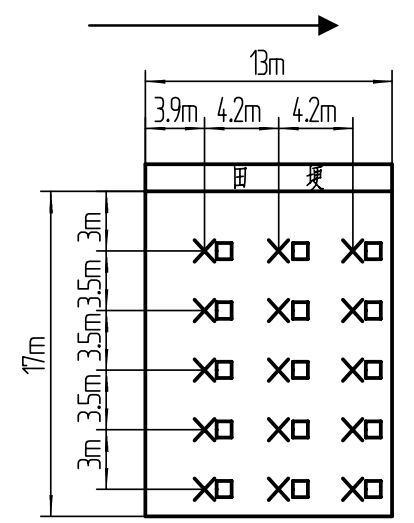

Fig. 4 Sample deployment of different spray guns

Among which: $\quad x-$ Is the legend for plant sample and

口— Is the legend for ground sample 


\section{Crop and Ground Sampling.}

- First, the experiment assessed the planting holes in the corresponding experimental fields. Knowing the area of each experimental field and the conditions of the planting holes, it then determined the number of plants that needed to be sampled at each point.

- During the later stage of growth, in order to facilitate the measurement of the pesticide solution's sediment distribution on different parts of the crop, entire rice plants were sampled, divided into upper, middle, and lower parts, and put into separate bags for testing and analysis.

- Sampling rice crops involved the deployment of samples in each group along the spray trajectory. Each experimental field deployed no less than three groups of samples and, as per spray gun flow rates and different ranges, deployed at lease 5-6 points along the spray trajectory in order to gather statistical information and construct graphs for further study.

- The method of ground sample deployment was the same as that of crop sampling. Samples were deployed along the spray trajectory and culture dishes were used to collect the pesticide droplets on the ground. The area of the culture dish's opening was $135 \mathrm{~cm}^{2}$.

Sample Measurement. As per the requirements of study and analysis, elute the crop samples and ground samples with a certain quantity of clean water (the water quality was the same as that used in the preparation of the spray solution). When the volume of the elution solution was sufficiently constant, use the made-in-China V-5100 spectrophotometer to measure individually its absorbance, calculate the content and sediment ratio of "Lichunhong" aqueous solution on different parts of the rice crop, and determine the distribution ratio of "Lichunhong" aqueous solution on rice crops, in soil, and from drift loss.

\section{Experiment Results and Analysis}

High-Efficiency, Wide-Coverage and Long-Range Power Sprayers Configured With Different Spray Guns Sprayed Pesticide Solution on Rice Crops. See Table 1 For The Experimental Results of Sediment Distribution.

Table 1. Measurement table of pesticide solution sediment distribution on rice during its later growth stage.

\begin{tabular}{|c|c|c|c|c|c|}
\hline \multicolumn{6}{|c|}{ Experiment Conditions } \\
\hline \multirow{2}{*}{\multicolumn{3}{|c|}{ Equipment Used in Experiment }} & \multicolumn{3}{|c|}{ High-efficiency, wide-coverage, long-range power sprayers } \\
\hline & & & PQ20 spray gun & PQ30 spray gun & PQ40 spray gun \\
\hline \multicolumn{3}{|c|}{ Crops Used in Experiment } & Full heading stage of rice & $\begin{array}{l}\text { Full heading stage of } \\
\text { rice }\end{array}$ & $\begin{array}{l}\text { Full heading stage of } \\
\text { rice }\end{array}$ \\
\hline \multicolumn{3}{|c|}{ Concentration of applied solution (ppm) } & 4000 & 4000 & 4000 \\
\hline \multicolumn{3}{|c|}{ Quantity of applied solution (L/mu) } & 18.5 & 34.84 & 49.49 \\
\hline \multicolumn{3}{|c|}{ Planting density (number of plants $/ \mathrm{m}^{2}$ ) } & 525.6 & 524.2 & 523.2 \\
\hline \multicolumn{3}{|c|}{ Plant height $(\mathrm{cm})$} & 81 & 81 & 81 \\
\hline \multicolumn{3}{|c|}{ Sampling method } & \multicolumn{3}{|c|}{ Sampled from each of the upper, middle, and lower parts } \\
\hline \multicolumn{6}{|c|}{ Measurement results of pesticide solution sediment distribution } \\
\hline \multirow{3}{*}{$\begin{array}{l}\text { Distribution ratio of } \\
\text { pesticide solution on rice } \\
\text { plants }(\%)\end{array}$} & \multicolumn{2}{|c|}{ Upper } & 70.58 & 66.54 & 61.96 \\
\hline & \multicolumn{2}{|c|}{ Middle } & 18.95 & 21.92 & 24.28 \\
\hline & \multicolumn{2}{|c|}{ Lower } & 10.47 & 11.54 & 13.76 \\
\hline \multirow{4}{*}{$\begin{array}{l}\text { Rice plant pesticide } \\
\text { solution sedimentation } \\
\text { rate }(\mathrm{ml} / \mathrm{g})\end{array}$} & \multirow{3}{*}{ Plant } & Upper & $6.89 \times 10^{-3}$ & $8.54 \times 10^{-3}$ & $11.33 \times 10^{-3}$ \\
\hline & & Middle & $1.42 \times 10^{-3}$ & $4.93 \times 10^{-3}$ & $5.36 \times 10^{-3}$ \\
\hline & & Lower & $0.56 \times 10^{-3}$ & $2.14 \times 10^{-3}$ & $2.35 \times 10^{-3}$ \\
\hline & \multicolumn{2}{|c|}{ Soil } & $0.71 \times 10^{-3}$ & $1.69 \times 10^{-3}$ & $1.1 \times 10^{-3}$ \\
\hline \multirow{3}{*}{$\begin{array}{l}\text { Distribution ratio of } \\
\text { pesticide solution in rice } \\
\text { field }(\%)\end{array}$} & \multicolumn{2}{|c|}{ Plant } & 65.26 & 67 & 69.73 \\
\hline & \multicolumn{2}{|c|}{ Soil } & 18.98 & 21.8 & 19.37 \\
\hline & \multicolumn{2}{|c|}{ Drift loss } & 15.76 & 11.2 & 10.9 \\
\hline
\end{tabular}




\section{Analysis of Experimental Data.}

Sediment from the sprayed pesticide solution distributes in the rice field. The sedimentation ratio is highest on rice crops. Spraying with the High-efficiency, wide-coverage, long-range sprayers configured with the new PQ20, PQ30, and PQ40 long-range combination spray gun models, the sediment distribution ratio of pesticide solution in the rice field were as follows: On the rice plants: $65.26 \%, 67 \%$, and $69.73 \%$, On the soil: $18.98 \%, 21.8 \%$, and $19.37 \%$, From drift loss: $15.76 \%, 11.2 \%$, and $10.9 \%$ ( Fig. 5). As shown by the above, rice plants during its later growth stage received more than $65 \%$ of the pesticide solution. Since rice plants acquire dense, intersecting leaves with high surface index in its later growth stage, both the pesticide's adhesiveness to the leaf surface and its utilization factor increase.
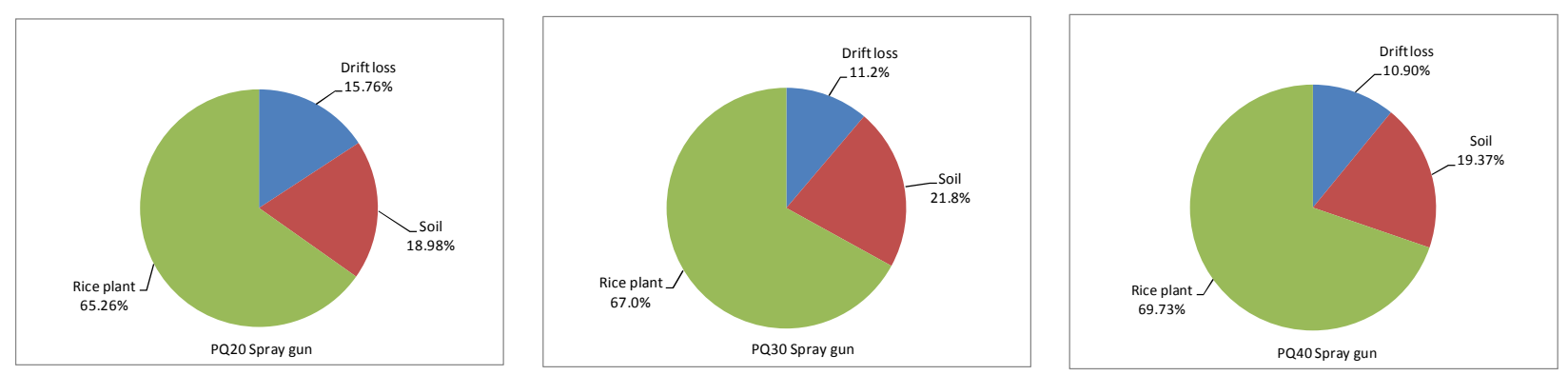

Fig. 5 Sediment distribution ratio of pesticide solution sprayed by different spray guns in rice fields.

The sediment distribution of pesticide spray at different parts of the crop significantly differed, sedimentation of pesticide solution mostly concentrated on the upper parts of the crop. Based on the sediment distribution ratio of pesticide solution in rice fields, the sediment distribution percentage of pesticide solution on rice crops is more than $65 \%$. But from the sedimentation ratio of pesticide solution on the upper, middle, and lower parts of the rice plants, it can be calculated that the sediment quantity received by the upper parts represented more than $60 \%$ of the total sediment quantity, the middle approximately $20 \%$, and the lower approximately $10 \%$. The main reason is during the late growth stage, rice enters into closure and most of the sprayed pesticide solution is first received by the leaves in the upper parts, resulting in quite significant sediment quantity. Spraying results from each of three kinds of spray guns demonstrate the same distribution pattern. The sediment distribution of the pesticide solution sprayed by different spray guns on different parts of the plants is shown in Fig. 6.

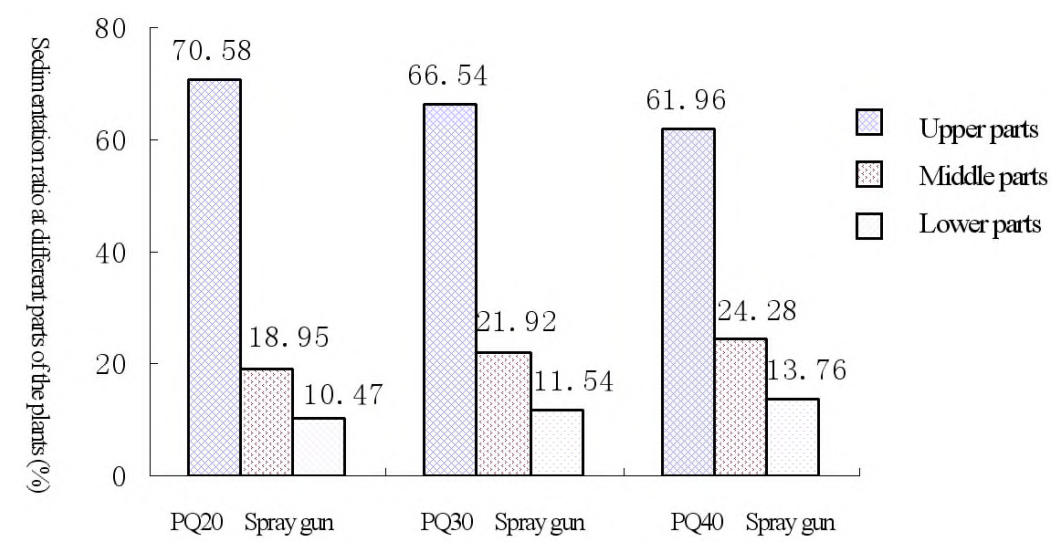

Fig. 6 Sedimentation ratio of pesticide solution on different parts of the plants.

Pesticide sedimentation on middle and lower parts of the crop improves with increase in spray gun flow rate. With the increase in spray gun flow rate, the diameter of the pesticide spray droplets gradually increases. Once the pesticide solution exits the spray gun, some small spray droplets fall to the upper leaves of the crop while most of the big spray droplets - broken down or 
pulled by gravity - roll or fall to the middle and lower parts of the crop to be received by the plant leaves, thereby increasing the sediment quantity of the pesticide solution on the middle and lower parts of the crop. As shown by the spraying results of the three configurations of spray guns (Fig. 6), sedimentation of the pesticide solution on lower and middle parts of the crop gradually increases with the increase in the spray gun's flow rate.

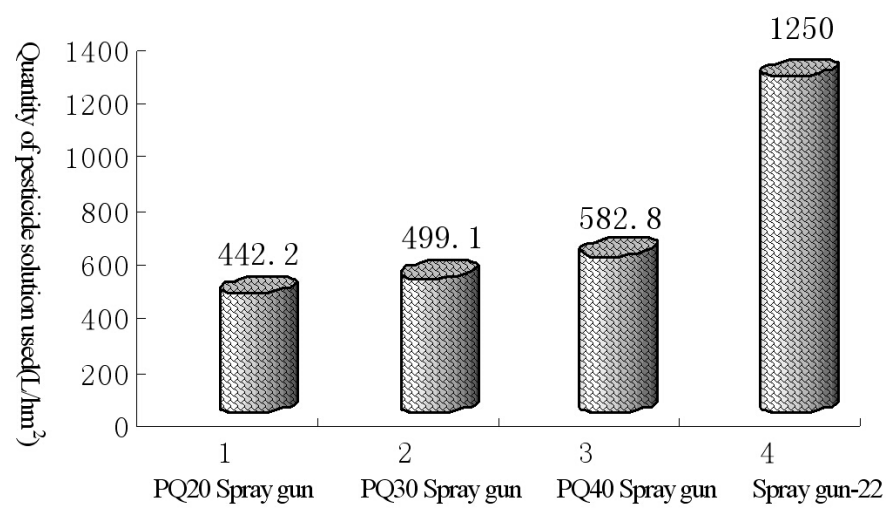

Fig. 7 Pesticide quantity by different spray guns

Compared with the old-style spray gun "Qiang-22", the high-efficiency, wide-coverage and long-range power sprayer configured with new spray gun models can significantly reduce the quantity of the pesticide solution used. Using the high-efficiency, wide-coverage and long-range power sprayer configured with the new spray gun, the $3 \mathrm{WKY}-40$ Model, the quantity of the pesticide solution used is $442.2-582.8 \mathrm{~L} / \mathrm{hm}^{2}$, which conserves more than $50 \%$ of pesticide solution compared to the Gongnong-36 power sprayer with the configuration of the "Qiang-22" spray gun (Fig. 7). Additionally, the experiment shows that the sediment distribution ratio of the pesticide solution on the crop has increased by multiple folds. It clearly demonstrates that reducing dosage can not only reduce solution loss and improve the utilization factor of the pesticide, but also reduce the quantity of agricultural chemicals used, conserve water resources, and promote work efficiency, thereby creating remarkable social and economic benefits.

\section{Conclusion}

The high-efficiency, wide-coverage and long-range power sprayer adopted the high-pressure, even-spraying technology. Its spray droplets have high penetrability, significantly increasing the sediment quantity of pesticide solution on the lower parts of rice. Furthermore, it effectively enhances the disease and pest prevention and control results in the middle and lower parts of rice and provides a satisfactory solution to the existing problems. High-efficiency, wide-coverage and long-range power sprayer and high-efficiency pesticide application methods will see more widespread promotion and application.

\section{Acknowledgements}

This research was supported by the National Natural Science Found of China (31401296) and the Independent Innovation Found of Science and Technology in Jiangsu Province (CX(14)2101).

\section{References}

[1] Y. Gong, X. M. Fu, P. Wu, The Analysis and Application of High-Efficiency, Wide-Coverage and Long-Range Sprayer in Paddy Protection, J. Farm Mach. 32 (2008) 46-47.

[2] X. M. Fu, P. Wu et al. Problem and Counter measures of Mechanical Control in Rice Produce Standardization, Chinese Agric. Mech. 5 (2006) 32-35. 
[3] P. Wu et al. The study of Pesticide Deposition Distribution of Common Spray Equipment and Application Method during the Later Stage of Rice Growth, Proceeding of Farming Machine Association, 1996, 136-139. 